\title{
A time-domain model of transient acoustic wave propagation in double-layered porous media
}

\author{
Z. E. A. Fellah and A. Wirgin \\ Laboratoire de Mécanique et d'Acoustique, CNRS-UPR 7051, 31 chemin Joseph Aiguier, Marseille, \\ 13009, France \\ M. Fellah \\ Laboratoire de Physique Théorique, Institut de Physique, USTHB, BP 32 El Alia, Bab Ezzouar 16111, \\ Algeria \\ N. Sebaa and C. Depollier \\ Laboratoire d'Acoustique de l'Université du Maine, UMR-CNRS 6613, Université du Maine, \\ Avenue Olivier Messiaen, 72085 Le Mans Cedex 09, France \\ W. Lauriks \\ Laboratorium voor Akoestiek en Thermische Fysica, Katholieke Universiteit Leuven, Celestijnenlaan 200 D, \\ B-3001 Heverlee, Belgium
}

\begin{abstract}
This paper concerns a time-domain model of transient wave propagation in double-layered porous materials. An analytical derivation of reflection and transmission scattering operators is given in the time domain. These scattering kernels are the medium's responses to an incident acoustic pulse. The expressions obtained take into account the multiple reflections occurring at the interfaces of the double-layered material. The double-layered porous media consist of two slabs of homogeneous isotropic porous materials with a rigid frame. Each porous slab is described by a temporal equivalent fluid model, in which the acoustic wave propagates only in the fluid saturating the material. In this model, the inertial effects are described by the tortuosity; the viscous and thermal losses of the medium are described by two susceptibility kernels which depend on the viscous and thermal characteristic lengths. Experimental and numerical results are given for waves transmitted and reflected by double-layered porous media formed by air-saturated plastic foam samples.
\end{abstract}

\section{INTRODUCTION}

The ultrasonic characterization of porous materials saturated by air ${ }^{1,2}$ is of great interest for a large class of industrial applications. These materials are frequently used in the automotive and aeronautics industries and in the building trade.

Ultrasonic characterization of materials is often achieved by measuring the attenuation coefficient and phase velocity in the frequency domain, ${ }^{3,4}$ or by solving the direct and inverse problems directly in the time domain. ${ }^{5-13}$ In the frequency domain, measurements of the attenuation coefficient may be more robust than measurements of phase velocity. In these situations, the application of the Kramers-Kronig ${ }^{11-13}$ dispersion relations may allow the determination of the phase velocity from the measured attenuation coefficient.

Many applications, such as medical imaging or inverse scattering, ${ }^{14}$ require a study of the behavior of pulses traveling into porous media. ${ }^{3,4,8-11}$ When a broadband ultrasound pulse passes through a layer of a medium, the pulse waveform changes as a result of attenuation and dispersion of the medium. The classic method for predicting a change in the waveform of a signal passing through a medium relies on the system's impulse response. According to the theory of linear systems, ${ }^{15}$ the output signal is a convolution of the input signal and the system impulse response. Many media, including porous materials and soft tissues, have been observed to have an attenuation function that increases with frequency ${ }^{16}$ As a result, higher frequency components of the pulse are attenuated more than lower frequency components. After passing through the layer, the transmitted pulse is not just a scaled-down version of the incident pulse, but has a different shape. Dispersion refers to the phenomenon observed when the phase velocity of a propagating wave changes with frequency. ${ }^{17}$ Dispersion causes the propagating pulse waveform to change because wave components with different frequencies travel at different speeds. An understanding of the interaction of ultrasound with a porous medium in both the time and frequency domains, and the ability to determine the change of waveform when propagating ultrasound pulses, should be useful in designing array transducers and in quantitative ultrasound tissue characterization. ${ }^{18,19}$

This time-domain model is an alternative to the classical frequency-domain approach. ${ }^{1,3,4}$ It is an advantage of the time domain that the results are immediate and direct. ${ }^{5-13}$ The attractive feature of a time-domain-based approach is that the analysis is naturally bounded by the finite duration of ultrasonic pressures, and is consequently the most appropri- 
ate approach for the transient signal. However, for wave propagation generated by time-harmonic incident waves and sources (monochromatic waves), the frequency analysis is more appropriate. ${ }^{1}$ A time-domain approach differs from frequency analysis in that the susceptibility functions describing viscous and thermal effects are convolution operators acting on velocity and pressure, and therefore a different algebraic formalism must be applied to solve the wave equation. The time-domain response of the material is described by an instantaneous response and a "susceptibility" kernel responsible for memory effects.

In the past, many authors have used fractional calculus ${ }^{20}$ as an empirical method to describe the properties of viscoelastic materials, e.g., see Caputo ${ }^{21}$ and Bagley and Torvik. ${ }^{22}$ The observation that asymptotic expressions of stiffness and damping in porous materials are proportional to the fractional powers of frequency ${ }^{23}$ suggests that time derivatives of a fractional order might describe the behavior of sound waves in this kind of material, including relaxation and frequency dependence. In this work, fractional calculus is used to describe viscous and thermal interaction between the fluid and the structure in double-layered porous media consisting of two slabs of homogeneous porous materials. Given the medium's response to an incident pulse, reflection and transmission scattering operators are calculated for double-layered porous media. Experimental results are compared with theoretical predictions, giving good correlation.

The outline of this paper is as follows. Section II shows a temporal equivalent fluid model, the connection between the fractional derivatives and the wave propagation in rigid porous media in high-frequency range is established, and the basic equations are written in the time domain. Sections III and IV are devoted to formulating the problem and analytical derivation of the reflection and transmission scattering kernels for double-layered porous media consisting of two slabs of homogeneous porous materials. The scattering responses of the media take into account the multiple reflections at the double-layered porous media interfaces. Finally, in Sec. V, experimental validation using ultrasonic measurement in transmission and reflection is discussed for air-saturated industrial plastic foams.

\section{TEMPORAL EQUIVALENT FLUID MODEL}

The quantities involved in sound propagation in porous materials can be defined locally, on a microscopic scale. However, this study is generally difficult because of the complicated frame geometries. Only the mean values of the quantities involved are of practical interest. Averaging must be performed on a macroscopic scale, using volumes with large enough dimensions for the average to be significant. At the same time, these dimensions must be much smaller than the wavelength. Even on a macroscopic scale, describing sound propagation in porous material can be very complicated, since sound also propagates in the frame of the material. If the frame is motionless, the porous material can be replaced on a macroscopic scale by an equivalent fluid.

In porous material acoustics, a distinction can be made between two situations depending on whether the frame is moving or not. In the first case, the wave dynamics due to coupling between the solid frame and the fluid is clearly described by the Biot theory. ${ }^{24,25}$ In air-saturated porous media, the structure is generally motionless and the waves propagate only in the fluid. This case is described by the equivalent fluid model which is a particular case in the Biot model, in which fluid-structure interactions are taken into account by the viscous susceptibility kernel, $\chi_{v}$, and the thermal susceptibility kernel, $\chi_{t h}$, as follows: ${ }^{8,26}$

$$
\begin{gathered}
\rho_{f} \alpha_{\infty} \partial_{t} \mathbf{v}(\mathbf{r}, t)+\int_{0}^{t} \chi_{v}\left(t-t^{\prime}\right) \partial_{t} \mathbf{v}\left(\mathbf{r}, t^{\prime}\right) d t^{\prime}=-\nabla p(\mathbf{r}, t), \\
\frac{1}{K_{a}} \partial_{t} p(\mathbf{r}, t)+\int_{0}^{t} \chi_{t h}\left(t-t^{\prime}\right) \partial_{t} p\left(\mathbf{r}, t^{\prime}\right) d t^{\prime}=-\nabla \mathbf{v}(\mathbf{r}, t) .
\end{gathered}
$$

Constitutive relations in the time domain result from arguments based on invariance under time translation and causality. ${ }^{11-13}$ In these equations, $p$ is the acoustic pressure, $\mathbf{v}$ is the particle velocity, $\rho_{f}$ and $K_{a}$ are the density and compressibility modulus of the fluid, respectively. The parameter $\alpha_{\infty}$ reflects the instantaneous response of the porous medium and describes the inertial coupling between fluid and structure. Instantaneous therefore means that the response time is much shorter than the typical time scale for acoustic field variation. The susceptibility kernels $\chi_{v}$ and $\chi_{t h}$ are memory functions which determine the dispersion of the medium.

The medium varies with depth $x$ only, and the incident wave is planar and normally incident. With no lack of generality, the pressure acoustic field can be assumed to have only one component, denoted $p(x, t)$. It is assumed that the pressure field in the medium is zero prior to $t=0$. The wave equation for the pressure acoustic field of a porous dispersive medium with a rigid frame is obtained from the constitutive equations (1) and (2), and is in the form

$$
\begin{gathered}
\partial_{x}^{2} p(x, t)-\frac{1}{c_{0}^{2}}\left[\alpha_{\infty} \partial_{t}^{2} p(x, t)+\left(\alpha_{\infty} K_{a} \chi_{t h}+\frac{\chi_{v}}{\rho_{f}}\right.\right. \\
\left.\left.+c_{0}^{2} \chi_{t h} * \chi_{v}\right) * \partial_{t}^{2} p(x, t)\right]=0,
\end{gathered}
$$

where $c_{0}=\left(K_{a} / \rho_{f}\right)^{1 / 2}$ is the speed of free fluid. The following notation is used for the convolution integral:

$$
[f * g](x, t)=\int_{0}^{t} f\left(x, t-t^{\prime}\right) g\left(x, t^{\prime}\right) d t^{\prime} .
$$

Viscous and thermal exchanges between a fluidsaturated porous medium and its structure are responsible for acoustic field damping. In the asymptotic regime, corresponding to high-frequency limit, ${ }^{8,26}$ viscous and thermal interactions are modeled by the memory relaxation operators $\chi_{v}(t)$ and $\chi_{t h}(t)$ given $b^{26}$

$$
\chi_{v}(t)=\frac{2 \rho_{f} \alpha_{\infty}}{\Lambda} \sqrt{\frac{\eta}{\pi \rho_{f}}} t^{-1 / 2},
$$




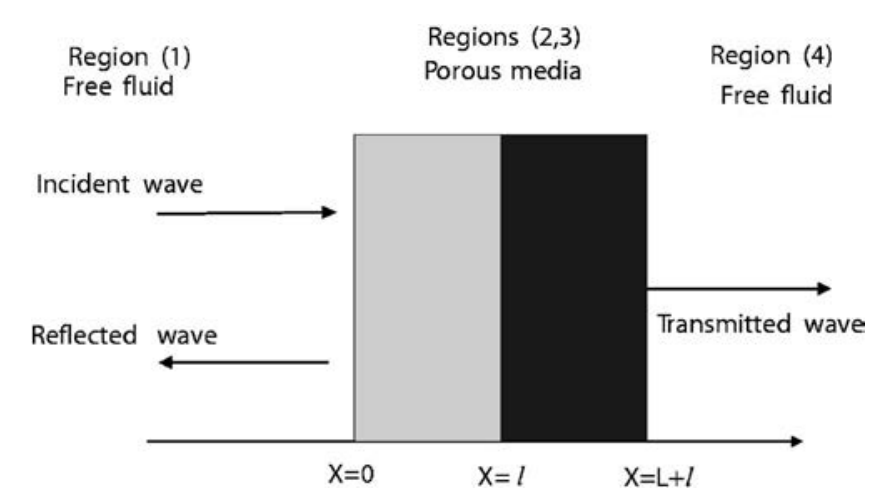

FIG. 1. Problem geometry.

$$
\chi_{t h}(t)=\frac{2(\gamma-1)}{K_{a} \Lambda^{\prime}} \sqrt{\frac{\eta}{\pi P_{r} \rho_{f}}} t^{-1 / 2},
$$

where $P_{r}$ is the Prandtl number, $\eta$ and $\gamma$ are the fluid viscosity and adiabatic constant, respectively. The model's relevant physical parameters are tortuosity $\alpha_{\infty}$, and viscous and thermal characteristic lengths, $\Lambda$ and $\Lambda^{\prime}$. In this model the time convolution of $t^{-1 / 2}$ with a function is interpreted as a semiderivative operator according to the definition of the fractional derivative of order $\nu$ given ${ }^{27}$ by

$$
D^{\nu}[x(t)]=\frac{1}{\Gamma(-\nu)} \int_{0}^{t}(t-u)^{-\nu-1} x(u) d u,
$$

where $\Gamma(x)$ is the gamma function.

\section{FORMULATING THE PROBLEM}

Consider a double-layered porous medium consisting of two homogeneous slabs with different acoustic parameters. The geometry of the problem is shown in Fig. 1. The first porous slab occupies the region $0 \leqslant x \leqslant \ell$ and the second one occupies the region $\ell \leqslant x \leqslant L$. Each porous slab is assumed to be isotropic and to have a rigid frame. A short sound pulse impinges normally on the medium from the left [free fluidregion (1)]. It gives rise to an acoustic pressure field $p_{i}(x, t)$, $i=2,3$ and an acoustic velocity field $v_{i}(x, t), i=2,3$ within each layer [the symbol $i=2,3$ denotes regions (2) and (3), respectively] which satisfy the propagation equation (3).

It is assumed that the pressure field is continuous at the boundary of each layer

$$
\begin{aligned}
& p\left(0^{+}, t\right)=p\left(0^{-}, t\right), \quad p\left(\ell^{-}, t\right)=p\left(\ell^{+}, t\right), \\
& p\left[(\ell+L)^{-}, t\right]=p\left[(\ell+L)^{+}, t\right]
\end{aligned}
$$

(where \pm superscript denotes the limit from left and right, respectively) and the initial conditions

$$
\left.p(x, t)\right|_{t=0}=\left.0 \quad \frac{\partial p}{\partial t}\right|_{t=0}=0,
$$

which mean that the medium is idle for $t=0$.

If the incident sound wave is launched in region $x \leqslant 0$ [region (1)], then the pressure field in the region on the left of the double-layered material is expressed as the sum of the incident and reflected fields

$$
p_{1}(x, t)=p^{i}\left(t-\frac{x}{c_{0}}\right)+p^{r}\left(t+\frac{x}{c_{0}}\right), \quad x<0,
$$

where $p_{1}(x, t)$ is the field in region $x<0, p^{i}$ and $p^{r}$ denote the incident and reflected fields, respectively. A transmitted field is also produced in the region on the right of the doublelayered material. This takes the form

$$
p_{4}(x, t)=p^{t}\left(t-\frac{\ell}{c_{2}}-\frac{L}{c_{3}}-\frac{x-\ell-L}{c_{0}}\right), \quad x>\ell+L .
$$

[ $p_{4}(x, t)$ is the field in region (4): $x>\ell+L$ and $p^{t}$ the transmitted field]. $c_{2}$ and $c_{3}$ represent the acoustic velocities in regions (2) and (3), respectively, defined by the relation $c_{i}$ $=c_{0} /\left(\alpha_{i}\right)^{1 / 2}, i=2,3$, where $\alpha_{i}$ represents the tortuosity of each porous layer.

The incident and scattered fields are related by scattering operators (i.e., reflection and transmission operators) for the material. These are integral operators represented by

$$
\begin{aligned}
p^{r}(x, t) & =\int_{0}^{t} \tilde{R}(\tau) p^{i}\left(t-\tau+\frac{x}{c_{0}}\right) d \tau \\
& =\widetilde{R}(t) * p^{i}(t) * \delta\left(t+\frac{x}{c_{0}}\right), \\
p^{t}(x, t) & =\int_{0}^{t} \tilde{T}(\tau) p^{i}\left(t-\frac{\ell}{c_{2}}-\frac{L}{c_{3}}-\frac{x-\ell-L}{c_{0}}\right) d \tau \\
& =\widetilde{T}(t) * p^{i}(t) * \delta\left(t-\frac{\ell}{c_{2}}-\frac{L}{c_{3}}-\frac{x-\ell-L}{c_{0}}\right),
\end{aligned}
$$

where $\delta(t)$ is the Dirac distribution. In Eqs. (12) and (13) the functions $\widetilde{R}$ and $\widetilde{T}$ are the respective reflection and transmission kernels for incidence from the left. Note that the lower limit of integration in (12) and (13) is set to 0, which is equivalent to assuming that the incident wavefront first impinges on the material at $t=0$. The operators $\widetilde{R}$ and $\widetilde{T}$ are independent of the incident field used in the scattering experiment and depend only on the properties of the materials.

In region $x \leqslant 0$, the field $p_{1}(x, t)$ is given by

$$
p_{1}(x, t)=\left[\delta\left(t-\frac{x}{c_{0}}\right)+\widetilde{R}(t) * \delta\left(t+\frac{x}{c_{0}}\right)\right] * p^{i}(t) .
$$

To simplify the analysis, we will use the Laplace transform which is appropriate for our problem. We note $\widetilde{P}_{i}(x, z), i$ $=1,2,3,4$, the Laplace transform of $p_{i}(x, t), i=1,2,3,4$ defined by

$$
\widetilde{P}_{i}(x, z)=\mathcal{L}\left[p_{i}(x, t)\right]=\int_{0}^{\infty} \exp (-z t) p_{i}(x, t) d t .
$$

The Laplace transform of the field outside the doublelayered medium is given by

$$
\tilde{P}_{1}(x, z)=\left[\exp \left(-z \frac{x}{c_{0}}\right)+R(z) \exp \left(z \frac{x}{c_{0}}\right)\right] \varphi(z), \quad x \leqslant 0,
$$




$$
\begin{gathered}
\widetilde{P}_{4}(x, z)=T(z) \exp \left[-\left(\frac{\ell}{c_{2}}+\frac{L}{c_{3}}+\frac{x-\ell-L}{c_{0}}\right) z\right] \varphi(z), \\
x \geqslant \ell+L,
\end{gathered}
$$

Here, $\widetilde{P}_{1}(x, z)$ and $\widetilde{P}_{4}(x, z)$ are the Laplace transform of the field on the left and right of the double-layered porous media, respectively, $\varphi(z)$ denotes the Laplace transform of the incident field $p^{i}(t)$, and finally $R(z)$ and $T(z)$ are the Laplace transforms of the reflection and transmission kernels, respectively.

The acoustic pressure fields $p_{i}(x, t), i=2,3$ inside each layer of the porous media [regions (2) and (3)] satisfy the propagation equation (3), which can be written in the Laplace domain as

$$
\frac{\partial^{2} \widetilde{P}_{i}(x, z)}{\partial x^{2}}-\frac{f_{i}(z)}{c_{i}^{2}} \widetilde{P}_{i}(x, z)=0, \quad i=2,3, \quad 0 \leqslant x \leqslant \ell+L .
$$

The function $f_{i}(z)$ is given by the following expression:

$$
f_{i}(z)=z^{2} c_{i}^{2}\left[\rho_{f} \alpha_{i}+\tilde{\chi}_{v i}(z)\right] \cdot\left[1 / K a+\tilde{\chi}_{t h i}(z)\right], \quad i=2,3,
$$

where $\widetilde{\chi}_{v i}(z)$ and $\tilde{\chi}_{t h i}(z)$ represent the Laplace transform of $\chi_{v i}(t)$ and $\chi_{t h i}(t)$, respectively, and their expressions in the time domain are given by

$$
\begin{aligned}
& \chi_{v i}(t)=\frac{2 \rho_{f} \alpha_{i}}{\Lambda_{i}} \sqrt{\frac{\eta}{\pi \rho_{f}}} t^{-1 / 2}, \quad i=2,3 . \\
& \chi_{t h i}(t)=\frac{2(\gamma-1)}{K_{a} \Lambda_{i}^{\prime}} \sqrt{\frac{\eta}{\pi P_{r} \rho_{f}}} t^{-1 / 2}, \quad i=2,3 .
\end{aligned}
$$

$\Lambda_{i}$ and $\Lambda_{i}^{\prime}, i=2,3$ are the viscous and thermal characteristic lengths of each porous layer.

By developing expression (19), we obtain the following relation for $f_{i}(z)$ :

$$
\begin{gathered}
f_{i}(z)=z^{2}+2 \sqrt{\frac{\eta}{\rho}}\left(\frac{1}{\Lambda_{i}}+\frac{\gamma-1}{\sqrt{P_{r} \Lambda_{i}^{\prime}}}\right) z \sqrt{z}+\frac{4(\gamma-1) \eta}{\rho_{f} \Lambda_{i} \Lambda_{i}^{\prime} \sqrt{P_{r}}} z, \\
i=2,3 .
\end{gathered}
$$

The solution of Eq. (18) can be expressed by

$$
\begin{aligned}
\widetilde{P}_{i}(x, z)= & {\left[A_{i}(z) \exp \left(-\frac{\sqrt{f_{i}(z)}}{c_{i}} x\right)\right.} \\
& \left.+B_{i}(z) \exp \left(\frac{\sqrt{f_{i}(z)}}{c_{i}} x\right)\right] \varphi(z), \quad i=2,3,
\end{aligned}
$$

where coefficients $A_{i}(z)$ and $B_{i}(z)$ can be determined by the physical conditions at the boundary of each layer. This is given in the next section.

\section{REFLECTION AND TRANSMISSION SCATTERING OPERATORS}

To derive reflection and transmission coefficients, we use the continuity relations of the acoustic pressure fields [Eqs. (8)] given in the Laplace domain by

$$
\begin{array}{r}
\widetilde{P}_{1}\left(0^{-}, z\right)=\widetilde{P}_{2}\left(0^{+}, z\right), \quad \widetilde{P}_{2}\left(\ell^{-}, z\right)=\widetilde{P}_{3}\left(\ell^{+}, z\right), \\
\widetilde{P}_{3}\left[(\ell+L)^{-}, z\right]=\widetilde{P}_{4}\left((\ell+L)^{+}, z\right) .
\end{array}
$$

Using the expressions of the pressure fields in each layer [Eqs. (16), (17), and (23)] and the conditions (24), we obtain the followings relations for the coefficients $A_{i}(z)$ and $B_{i}(z)$, $i=2,3$, and the coefficients $R(z)$ and $T(z)$ :

$$
A_{2}(z)+B_{2}(z)=\widetilde{P}_{2}(0, z)=1+R(z),
$$

$$
\begin{gathered}
A_{2}(z) \exp \left(-\frac{\sqrt{f_{2}(z)}}{c_{2}} \ell\right)+B_{2}(z) \exp \left(\frac{\sqrt{f_{2}(z)}}{c_{2}} \ell\right) \\
=A_{3}(z) \exp \left(-\frac{\sqrt{f_{3}(z)}}{c_{3}} \ell\right)+B_{3}(z) \exp \left(\frac{\sqrt{f_{3}(z)}}{c_{3}} \ell\right), \\
A_{3}(z) \exp \left(-\frac{\sqrt{f_{3}(z)}}{c_{3}}(\ell+L)\right)+B_{3}(z) \exp \left(\frac{\sqrt{f_{3}(z)}}{c_{3}}(\ell+L)\right) \\
=T(z) \exp \left[-\left(\frac{\ell}{c_{2}}+\frac{L}{c_{3}}\right) z\right] .
\end{gathered}
$$

The Euler equation in each region is written as

$\rho_{f} \alpha_{i} \partial_{t} v_{i}(x, t)+\chi_{v i}(t) * \partial_{t} v_{i}(x, t)=-\partial_{x} p_{i}(x, t), \quad i=1, \ldots, 4$.

In these equations $v_{i}(x, t) i=1, \ldots, 4$ is the acoustic velocity field in regions (1),.., (4). Note that in regions (1) and (4), corresponding to the free fluid, porosity and tortuosity values are $1\left(\alpha_{1}=\alpha_{4}=1\right.$ and $\left.\phi_{1}=\phi_{4}=1\right)$, and the viscous susceptibility kernel vanishes $\left(\chi_{v i}=0, i=1,4\right)$ outside the doublelayered porous media.

The equation for flow continuity between each interface $(x=0, x=\ell$, and $x=\ell+L)$ is given by

$$
\phi_{i} v_{i}(x, t)=\phi_{i+1} v_{i+1}(x, t), \quad i=1,2,3,
$$

where $\phi_{i}, i=1, \ldots, 4$ is the porosity of each layer.

Using the relations (28) and (29), we obtain the following relations between the acoustic pressure $p_{i}(x, t)$ and physical properties of each layer:

$$
\begin{aligned}
& \phi_{i+1}\left[\rho_{f} \alpha_{i} \partial_{x} p_{i+1}(x, t)+\chi_{v i}(t) * \partial_{x} p_{i+1}(x, t)\right] \\
& \quad=\phi_{i}\left[\rho_{f} \alpha_{i+1} \partial_{x} p_{i}(x, t)+\chi_{v(i+1)}(t) * \partial_{x} p_{i}(x, t)\right], \quad i=1,2,3 .
\end{aligned}
$$

Using the Laplace transform of Eq. (30) and the pressure field expressions for each layer [Eqs. (16), (17), and (23)], we obtain the following relations at the interface of each layer:

$$
B_{2}(z)-A_{2}(z)=K_{1}(R(z)-1),
$$

$$
\begin{aligned}
& B_{3}(z) \exp \left(\frac{\sqrt{f_{3}(z)}}{c_{3}} \ell\right)-A_{3}(z) \exp \left(-\frac{\sqrt{f_{3}(z)}}{c_{3}} \ell\right) \\
& \quad=K_{2}\left[B_{2}(z) \exp \left(\frac{\sqrt{f_{2}(z)}}{c_{2}} \ell\right)-A_{2}(z) \exp \left(-\frac{\sqrt{f_{2}(z)}}{c_{2}} \ell\right)\right],
\end{aligned}
$$




$$
\begin{gathered}
B_{3}(z) \exp \left(\frac{\sqrt{f_{3}(z)}}{c_{3}}(\ell+L)\right)-A_{3}(z) \exp \left(-\frac{\sqrt{f_{3}(z)}}{c_{3}}(\ell+L)\right) \\
=K_{3} T(z) \exp \left[-\left(\frac{\ell}{c_{2}}+\frac{L}{c_{3}}\right) z\right],
\end{gathered}
$$

with

$$
K_{1}=\frac{\sqrt{\alpha_{2}}}{\phi_{2}}, \quad K_{2}=\frac{\phi_{2} \sqrt{\alpha_{3}}}{\phi_{3} \sqrt{\alpha_{2}}}, \quad K_{3}=\frac{\sqrt{\alpha_{3}}}{\phi_{3}}, \quad K_{1} K_{2}=K_{3} .
$$

Using the relations (25)-(27) and (31)-(33) (see Appendix A), we obtain the following expressions of the reflection and transmission coefficients:

$$
\begin{aligned}
& R(z)=d_{1} \frac{\Gamma(z)}{\Xi(z)}, \\
& T(z)=h_{1} \exp \left[\left(\frac{\ell}{c_{2}}+\frac{L}{c_{3}}\right) z\right] \frac{\exp \left(-2 \frac{\sqrt{f_{2}(z)}}{c_{2}} \ell-\frac{\sqrt{f_{3}(z)}}{c_{3}} L\right)}{\Xi(z)},
\end{aligned}
$$

with

$$
\begin{aligned}
\Gamma(z)= & 1+d_{2} \exp \left(-2 \frac{\sqrt{f_{2}(z)}}{c_{2}} \ell\right)+d_{3} \exp \left(-2 \frac{\sqrt{f_{3}(z)}}{c_{3}} L\right) \\
& -d_{4} \exp \left(-2 \frac{\sqrt{f_{2}(z)}}{c_{2}} \ell-2 \frac{\sqrt{f_{3}(z)}}{c_{3}} L\right), \\
\Xi(z)= & 1+h_{2} \exp \left(-2 \frac{\sqrt{f_{2}(z)}}{c_{2}} \ell\right)+h_{3} \exp \left(-2 \frac{\sqrt{f_{3}(z)}}{c_{3}} L\right) \\
& +h_{4} \exp \left(-2 \frac{\sqrt{f_{2}(z)}}{c_{2}} \ell-2 \frac{\sqrt{f_{3}(z)}}{c_{3}} L\right) . \\
d_{1}= & \frac{K_{1}-1}{K_{1}+1}, \quad d_{2}=\frac{\left(K_{1}+1\right)\left(K_{2}-1\right)}{\left(K_{1}-1\right)\left(K_{2}+1\right)}, \\
d_{3}= & \frac{\left(K_{3}-1\right)\left(K_{2}-1\right)}{\left(K_{3}+1\right)\left(K_{2}+1\right)}, \quad d_{4}=-\frac{\left(K_{3}-1\right)\left(K_{1}+1\right)}{\left(K_{3}+1\right)\left(K_{1}-1\right)}, \\
h_{1}= & \frac{4 K_{1} K_{2}}{\left(1+K_{3}\right)\left(1+K_{1}+K_{2}+K_{3}\right)}, \\
h_{2}= & \frac{\left(1-K_{2}\right)\left(1-K_{1}\right)}{\left(1+K_{1}\right)\left(1+K_{2}\right)}, \quad h_{3}=\frac{\left(K_{3}-1\right)\left(1-K_{2}\right)}{\left(K_{3}+1\right)\left(1+K_{2}\right)}, \\
h_{4}= & \left(1-K_{3}\right)\left(K_{1}-1\right) \\
\left(1+K_{3}\right)\left(K_{1}+1\right) &
\end{aligned}
$$

To express $n$-multiple reflections in porous layers, we shall write the reflection and transmission coefficients as follows:

$$
R(z)=d_{1} \Gamma(z) \sum_{n \geqslant 0}(-1)^{n}(\Xi(z)-1)^{n},
$$

$$
\begin{aligned}
T(z)= & h_{1} \exp \left[\left(\frac{\ell}{c_{2}}+\frac{L}{c_{3}}\right) z\right] \exp \left(-2 \frac{\sqrt{f_{2}(z)}}{c_{2}} \ell\right. \\
& \left.-\frac{\sqrt{f_{3}(z)}}{c_{3}}(L)\right) \sum_{n \geqslant 0}(-1)^{n}(\Xi(z)-1)^{n} .
\end{aligned}
$$

Using the identity

$$
(x+y+z)^{n}=\sum_{n_{1}+n_{2}+n_{3}=n} \frac{n !}{n_{1} ! n_{2} ! n_{3} !} x^{n_{1}} y^{n_{2}} z^{n_{3}},
$$

where $n !=\Gamma(n+1)$, the reflection and transmission expressions become

$$
\begin{aligned}
R(z)= & d_{1} \Gamma(z) \sum_{n \geqslant 0}(-1)^{n} n ! \sum_{n_{1}+n_{2}+n_{3}=n} \frac{h_{2}^{n_{1}} h_{3}^{n_{2}} h_{4}^{n_{3}}}{n_{1} ! n_{2} ! n_{3} !} \\
& \times \exp \left(-2 \frac{\sqrt{f_{2}(z)}}{c_{2}}\left(n_{1}+n_{3}\right) \ell\right) \\
& \times \exp \left(-2 \frac{\sqrt{f_{3}(z)}}{c_{3}}\left(n_{2}+n_{3}\right) L\right),
\end{aligned}
$$

and

$$
\begin{aligned}
T(z)= & h_{1} \exp \left[\left(\frac{\ell}{c_{2}}+\frac{L}{c_{3}}\right) z\right] \\
& \times \sum_{n \geqslant 0}(-1)^{n} n ! \sum_{n_{1}+n_{2}+n_{3}=n} \frac{h_{2}^{n_{1}} h_{3}^{n_{2}} h_{4}^{n_{3}}}{n_{1} ! n_{2} ! n_{3} !} \\
& \times \exp \left(-\frac{\sqrt{f_{2}(z)}}{c_{2}}\left(2 n_{1}+2 n_{3}+1\right) \ell\right) \\
& \times \exp \left(-\frac{\sqrt{f_{3}(z)}}{c_{3}}\left(2 n_{2}+2 n_{3}+1\right) L\right) .
\end{aligned}
$$

By setting $z=j \omega$, where $j^{2}=-1$ and $\omega$ is the angular frequency, we can easily deduce the expressions of the reflection and transmission coefficients in the frequency domain.

Recall that the inverse Laplace transforms of $\exp \left[-\left(\ell / c_{2}\right) \sqrt{f_{2}(z)}\right]$ and $\exp \left[-\left(L / c_{3}\right) \sqrt{f_{3}(z)}\right]$ are the Green function ${ }^{28}$ of the first and second porous slab, respectively.

In the time domain, the transmission scattering operator is expressed as

$$
\begin{aligned}
\widetilde{T}(t)= & h_{1} \sum_{n \geqslant 0}(-1)^{n} n ! \sum_{n_{1}+n_{2}+n_{3}=n} \frac{h_{2}^{n_{1}} h_{3}^{n_{2}} h_{4}^{n_{3}}}{n_{1} ! n_{2} ! n_{3} !} F_{2}\left[t+\frac{\ell}{c_{2}},\left(2 n_{1}\right.\right. \\
& \left.\left.+2 n_{3}+1\right) \frac{\ell}{c_{2}}\right] * F_{3}\left[t+\frac{L}{c_{3}},\left(2 n_{2}+2 n_{3}+1\right) \frac{L}{c_{3}}\right],
\end{aligned}
$$

where $F_{i}, i=2,3$ is the Green function ${ }^{28}$ of porous layers (2) and (3), respectively (see Appendix B).

The reflection scattering operator is expressed by the relation 


$$
\begin{aligned}
\tilde{R}(t)= & d_{1} \sum_{n \geqslant 0}(-1)^{n} n ! \sum_{n_{1}+n_{2}+n_{3}=n} \frac{h_{2}^{n_{1}} h_{3}^{n_{2}} h_{4}^{n_{3}}}{n_{1} ! n_{2} ! n_{3} !} \\
& \times\left[F_{2}\left[t, 2\left(n_{1}+n_{3}\right) \frac{\ell}{c_{2}}\right] * F_{3}\left[t, 2\left(n_{2}+n_{3}\right) \frac{L}{c_{3}}\right]\right. \\
& +d_{2} F_{2}\left[t, 2\left(n_{1}+n_{3}+1\right) \frac{\ell}{c_{2}}\right] * F_{3}\left[t, 2\left(n_{2}+n_{3}\right) \frac{L}{c_{3}}\right] \\
& +d_{3} F_{2}\left[t, 2\left(n_{1}+n_{3}\right) \frac{\ell}{c_{2}}\right] * F_{3}\left[t, 2\left(n_{2}+n_{3}+1\right) \frac{L}{c_{3}}\right] \\
& +d_{4} F_{2}\left[t, 2\left(n_{1}+n_{3}+1\right) \frac{\ell}{c_{2}}\right] \\
& \left.* F_{3}\left[t, 2\left(n_{2}+n_{3}+1\right) \frac{L}{c_{3}}\right]\right] .
\end{aligned}
$$

If only the first reflections at interfaces $x=0, x=\ell$ and $x=L$ are taken into account, the reflection scattering kernel expression becomes

$$
\begin{aligned}
\widetilde{R}(t)= & d_{1} \delta(t)+d_{1}\left(d_{2}-h_{2}\right) F_{2}\left[t, 2 \frac{\ell}{c_{2}}\right]+d_{1}\left(d_{4}-h_{4}-d_{2} h_{3}\right. \\
& \left.-d_{3} h_{2}+2 h_{2} h_{3}\right) F_{2}\left[t, 2 \frac{\ell}{c_{2}}\right] * F_{3}\left[t, 2 \frac{L}{c_{3}}\right] .
\end{aligned}
$$

The transmission scattering kernel describing the direct wave transmitted through two layers of porous materials without internal reflection is expressed by

$$
\begin{aligned}
\widetilde{T}(t) & =h_{1} F_{2}\left[t+\frac{\ell}{c_{2}}, \frac{\ell}{c_{2}}\right] * F_{3}\left[t+\frac{L}{c_{3}}, \frac{L}{c_{3}}\right] \\
& =h_{1} \int_{0}^{t} F_{2}\left[\tau+\frac{\ell}{c_{2}}, \frac{\ell}{c_{2}}\right] F_{3}\left[t-\tau+\frac{L}{c_{3}}, \frac{L}{c_{3}}\right] d \tau .
\end{aligned}
$$

The first term on the right-hand side of Eq. (44), $d_{1} \delta(t)$ $=\left[\left(\sqrt{\alpha_{2}-\phi_{2}}\right) /\left(\sqrt{\alpha_{2}+\phi_{2}}\right)\right] \delta(t)$, is equivalent to the instantaneous reflected response of the first layer (region 2). The part of the wave which is equivalent to this term corresponds to the wave reflected by the first interface $x=0$ of the first porous layer. It depends only on the porosity and tortuosity of the first porous slab. The wave reflected by the first interface has the advantage of not being dispersive, but simply attenuated by factor $d_{1}$. This result is in agreement with the conclusions obtained in other works for wave reflected by a slab of porous material. ${ }^{9,29,30}$ This shows that it is possible to measure the porosity and tortuosity of the first porous layer just by measuring its first reflected wave.

The second term on the right-hand side of Eq. (44), $d_{1}\left(d_{2}-h_{2}\right) F_{2}\left[t, 2\left(\ell / c_{2}\right)\right]$, corresponds to the second interface reflection contribution, $x=\ell$. This term depends on the porosity and tortuosity of the two porous layers. The Green's function $F_{2}$ describes the propagation and dispersion of an acoustic wave making one round trip inside the first porous slab. This Green's function depends on the viscous and thermal characteristics lengths $\Lambda$ and $\Lambda^{\prime}$ of the first layer (region 2). This result means that it can be possible to get information of all the acoustical properties (porosity, tortuosity, vis-

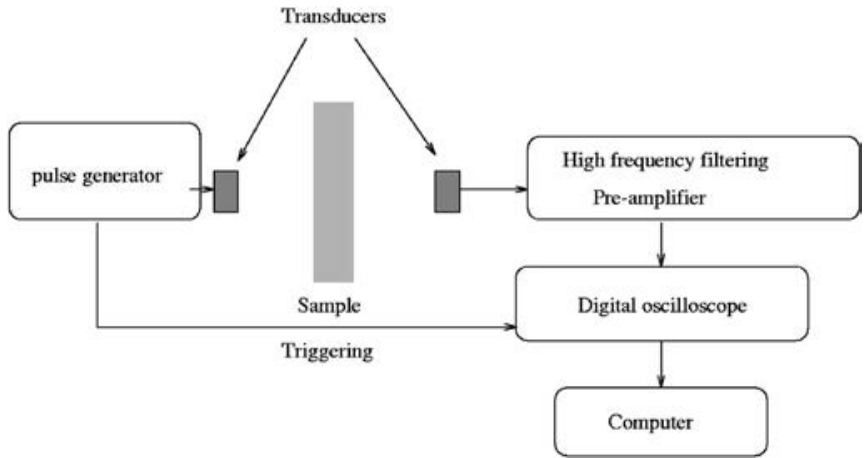

FIG. 2. Experimental setup of the ultrasonic measurements in transmitted mode.

cous and thermal characteristics lengths) of the first porous layer (region 2), and also, the porosity and tortuosity of the second porous layer (region 3), but the viscous and thermal characteristics lengths of the second porous layer (region 3) do not intervene.

Finally, the term $d_{1}\left(d_{4}-h_{4}-d_{2} h_{3}-d_{3} h_{2}\right.$ $\left.+2 h_{2} h_{3}\right) F_{2}\left[t, 2\left(\ell / c_{2}\right)\right] * F_{3}\left[t, 2\left(L / c_{3}\right)\right]$ represents the reflection contribution of the third interface, $x=L$. The corresponding wave makes one round trip inside the two porous layers. Evidently this wave contribution depends on all acoustical parameters of each porous layer.

The advantage of the obtained time-domain expression of the reflection and transmission scattering operators [Eqs. (44) and (45)] is to show analytically the effect of the acoustical parameters (porosity, tortuosity, viscous and thermal characteristic lengths) of each porous layer on the reflection contributions by the interfaces of the double-layered media.

\section{EXPERIMENTAL VALIDATION}

In application of this model, several numerical simulations for reflected and transmitted acoustic waves by two layered porous materials are compared to experimental data. Experiments are performed in the air using two broadband Ultran NCT202 transducers with a central frequency of $190 \mathrm{kHz}$ in air and a bandwidth of $6 \mathrm{~dB}$ from 150 to $230 \mathrm{kHz}$. Pulses of $400 \mathrm{~V}$ are provided by a 5052PR Panametrics pulser/receiver. The signals received are amplified to $90 \mathrm{~dB}$ and filtered above $1 \mathrm{MHz}$ to avoid high-

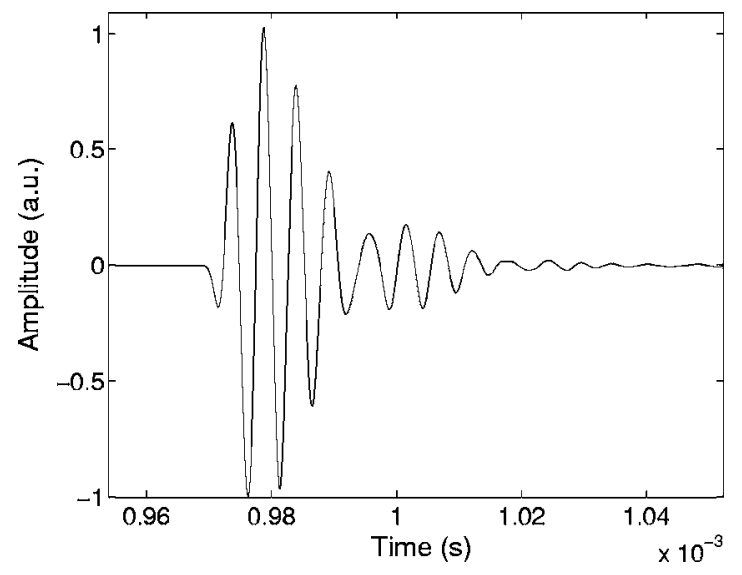

FIG. 3. Incident signal given out by the transducer in transmitted mode. 


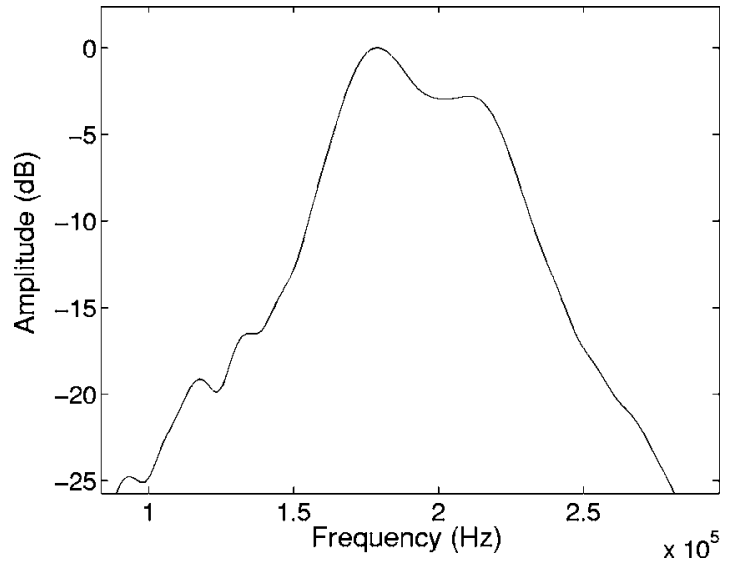

FIG. 4. Spectrum of the incident signal generated by the transducer in transmitted mode.

frequency noise (energy is totally filtered by the sample in this upper-frequency domain). Electronic interference is eliminated by 1000 acquisition averages. The experimental setup is shown in Fig. 2. The experimental incident signal generated by the transducer is given in Fig. 3. The amplitude is represented by an arbitrary unit (a.u.) and the point number represented in the abscissa is proportional to time. Signal duration is important as its spectrum must verify the condition of high-frequency approximation ${ }^{8,26}$ referred to in Sec. II. The spectrum of the incident signal is given in Fig. 4.

Measurements were made on plastic foam samples M1M4. Their acoustic characteristics were determined independently using classical methods ${ }^{8}$ (which were developed for a slab of porous material). The acoustical parameters of the plastic foam samples are given in Table I.

Three samples of double-layered porous materials were considered, the first consists of $0.86 \mathrm{~cm}$ of $\mathrm{M} 1$ and $0.81 \mathrm{~cm}$ of M2, the second of $4.13 \mathrm{~cm}$ of M1 and $1.99 \mathrm{~cm}$ of M3, and finally the third of $2.98 \mathrm{~cm}$ of M1 and $1.99 \mathrm{~cm}$ of M3. Numerical simulation and experimental results (transmitted signal) for the three samples of double-layered materials are presented in Figs. 5-7, respectively. The numerical results are obtained from convolution of the transmission operator [Eq. (45)] with the signal generated by the transducer shown in Fig. 3. The reader can see, from Figs. 5-7, the good correlation obtained between the experimental transmitted signal (solid line) and simulated signal (dashed line). This result validates the expression of the transmission scattering operator [Eq. (45)].

Reflected waves were processed by another experimental setup shown in Fig. 8. One transducer was used alternatively as a transmitter and receiver to detect the reflected

TABLE I. Acoustical characteristics of the plastic foams samples.

\begin{tabular}{ccccc}
\hline \hline Material & M1 & M2 & M3 & M4 \\
\hline Viscous characteristic length $[\Lambda(\mu \mathrm{m})]$ & 200 & 30 & 330 & 230 \\
Thermal characteristic length $\left[\Lambda^{\prime}(\mu \mathrm{m})\right]$ & 600 & 90 & 990 & 690 \\
Tortuosity $(\alpha)$ & 1.07 & 1.4 & 1.02 & 1.05 \\
Porosity $(\phi)$ & 0.97 & 0.85 & 0.90 & 0.98 \\
\hline \hline
\end{tabular}

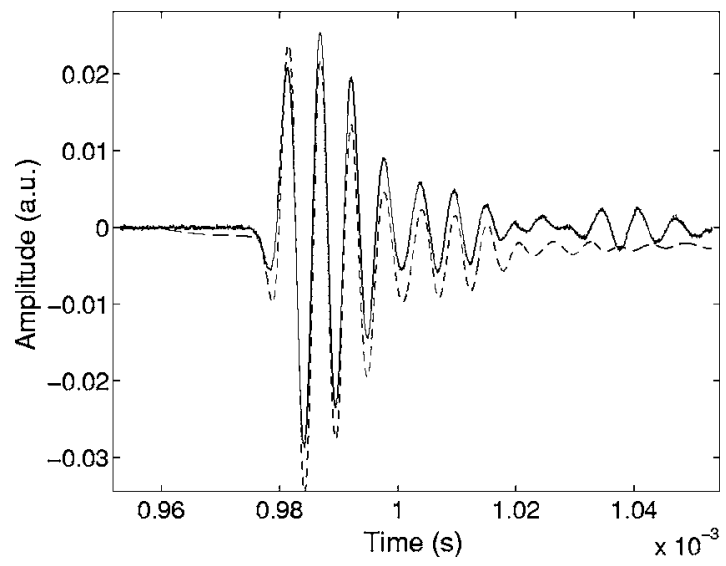

FIG. 5. Comparison between experimental transmitted signal (solid line) and simulated transmitted signal (dashed line) for a double-layered medium consisting of $0.86 \mathrm{~cm}$ of $\mathrm{M} 1$ and $0.81 \mathrm{~cm}$ of $\mathrm{M} 2$.

wave. The experimental incident signal used in reflected mode is given in Fig. 9 and its spectrum in Fig. 10.

A double-layered porous medium consisting of $1.11 \mathrm{~cm}$ of M4 and $0.87 \mathrm{~cm}$ of M1 was considered. Figure 11 shows a comparison between a simulated reflected signal (dashed line) and an experimental reflected signal (solid line). The simulated signal was obtained through convolution of the reflection scattering kernel given in Eq. (44) with the incident signal given in Fig. 9. Three reflected signals can be seen in Fig. 11. The first corresponds to the reflection of the first porous layer M4 $(x=0)$ by the first interface, this reflected wave corresponds to the first term on the right-hand side of Eq. (44): $d_{1} \delta(t)=\left[\left(\sqrt{\alpha_{2}}-\phi_{2}\right) /\left(\sqrt{\alpha_{2}}+\phi_{2}\right)\right] \delta(t)$. The second reflected wave given in Fig. 11 corresponds to the reflection between the second M4 interface and the first M1 interface $(x=\ell)$; this wave corresponds to the second term on the right-hand side of Eq. (44): $d_{1}\left(d_{2}-h_{2}\right) F_{2}\left[t, 2\left(\ell / c_{2}\right)\right]$. Finally, the third signal corresponds to reflection by the second M1 interface $(x=L)$, which is given by the term $d_{1}\left(d_{4}-h_{4}\right.$ $\left.-d_{2} h_{3}-d_{3} h_{2}+2 h_{2} h_{3}\right) F_{2}\left[t, 2\left(\ell / c_{2}\right)\right] * F_{3}\left[t, 2\left(L / c_{3}\right)\right]$. Generally, it is not possible to see the other reflection contributions experimentally because of the high damping of ultrasonic waves in air-saturated plastic foams. However, the third reflection contribution at $x=L$ is not always seen experimen-

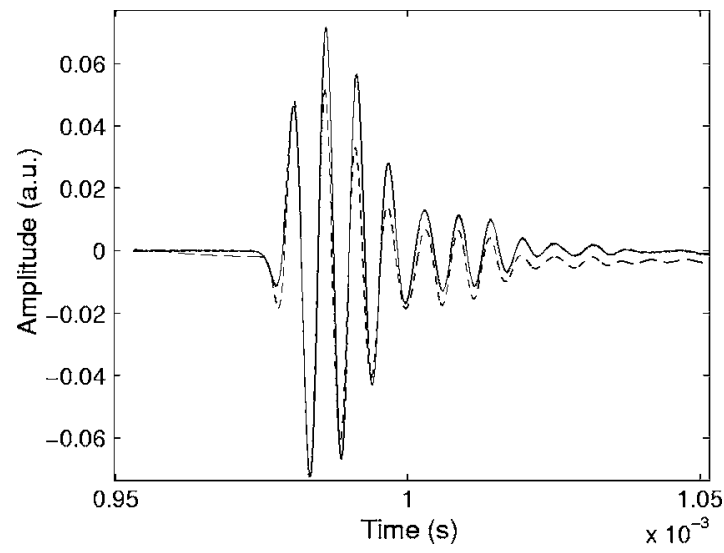

FIG. 6. Comparison between experimental transmitted signal (solid line) and simulated transmitted signal (dashed line) for a double-layered medium consisting of $4.13 \mathrm{~cm}$ of $\mathrm{M} 1$ and $1.99 \mathrm{~cm}$ of M3. 


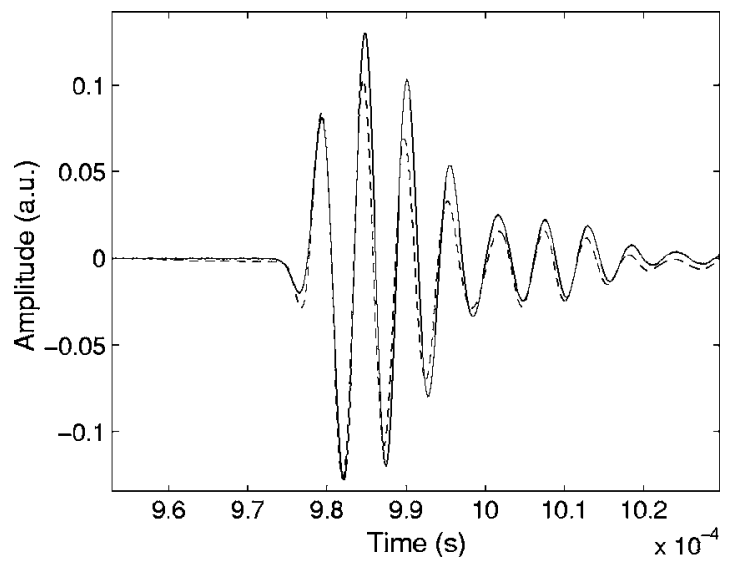

FIG. 7. Comparison between experimental transmitted signal (solid line) and simulated transmitted signal (dashed line) for a double-layered medium consisting of $2.98 \mathrm{~cm}$ of M1 and $1.99 \mathrm{~cm}$ of M3.

tally; for example, Fig. 12 shows a comparison between theoretical predictions (dashed line) and experimental results (solid line) for a double-layered medium consisting of $0.88 \mathrm{~cm}$ of $\mathrm{M} 1$ and $0.83 \mathrm{~cm}$ of M2. Acoustic attenuation in plastic foam sample M2 is higher than in the other samples. Sample M2 has high tortuosity and low characteristic lengths compared to those of the other plastic foam samples, which indicates high acoustic damping. In Fig. 12, we can only see the two reflected waves corresponding to reflection by the first M1 (first layer) interface $(x=0)$ and reflection between the second M1 interface and the first M2 (second layer) interface $(x=\ell)$, respectively. The reflected wave of the second M2 interface $(x=L)$ is fully absorbed by the two layers, M1 and M2. We can also see in Fig. 12 that the amplitude of the second reflected wave is greater than that of the first reflected wave. This is due to the high resistivity of sample M2 near sample M1 and the thickness of M1, which also plays an important part in attenuating the wave reflected at the second interface, $x=\ell$.

\section{CONCLUSION}

In this paper the analytical expressions of reflection and transmission scattering operators are derived for double-

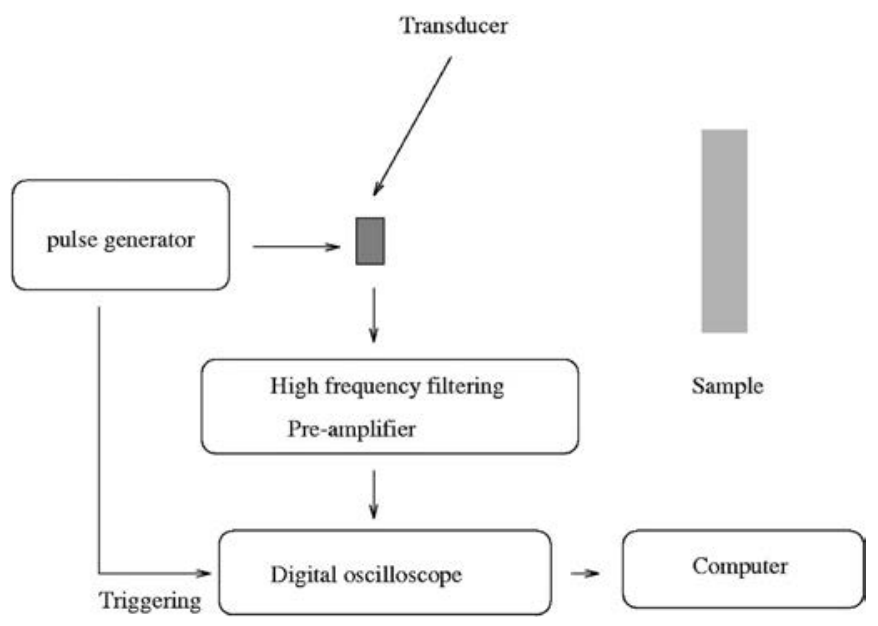

FIG. 8. Experimental setup of the ultrasonic measurements in reflected mode.

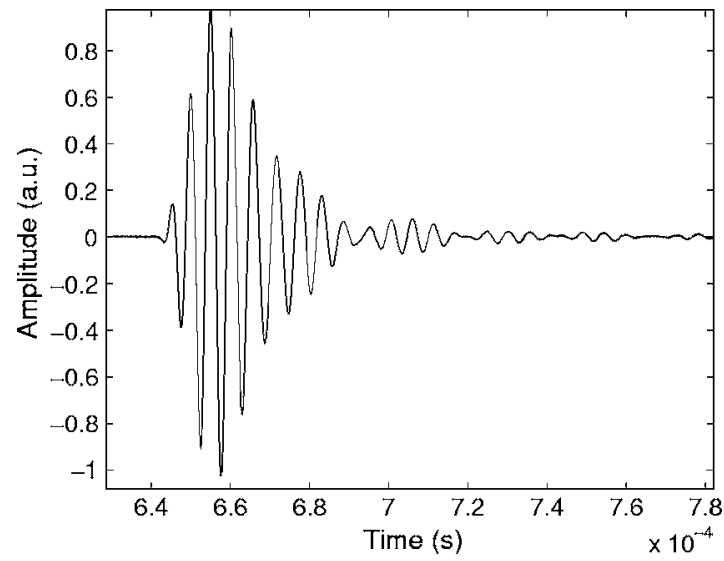

FIG. 9. Incident signal given out by the transducer in reflected mode.

layered porous media consisting of two homogeneous isotropic materials. Simple relationships are given between these operators and the acoustic parameters of the medium. It is shown that the scattering operators are equal to the sum of the contribution of each interface to the double-layered porous medium. The advantages of the analytical expressions of reflection and transmission scattering operators in the time domain is to show easily the effect of the acoustical parameters on the multiple reflections at the interfaces of the double-layered medium.

Ultrasonic measurements in the transmission and reflection mode were processed using different experimental setups. A slight difference was observed between theoretical predictions and experimental data in the two modes (reflection and transmission). This leads to the conclusion that the expressions of scattering operators obtained are correct. Future studies will concentrate on the inverse problem, and methods and inversion algorithms will be developed to optimize the acoustic properties of double-layered air-saturated porous media for reflected and transmitted ultrasonic waves.

\section{APPENDIX A: EXPRESSION OF THE REFLECTION AND TRANSMISSION OPERATORS}

Using Eqs. (25) and (31), we can write the following equation system given the relations between $A_{2}(z), B_{2}(z)$, and $R(z)$ :

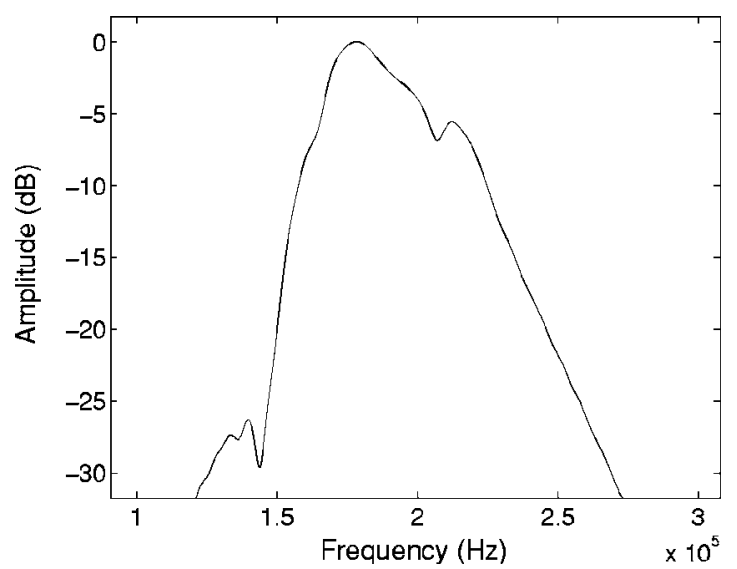

FIG. 10. Spectrum of the incident signal generated by the transducer in reflected mode. 


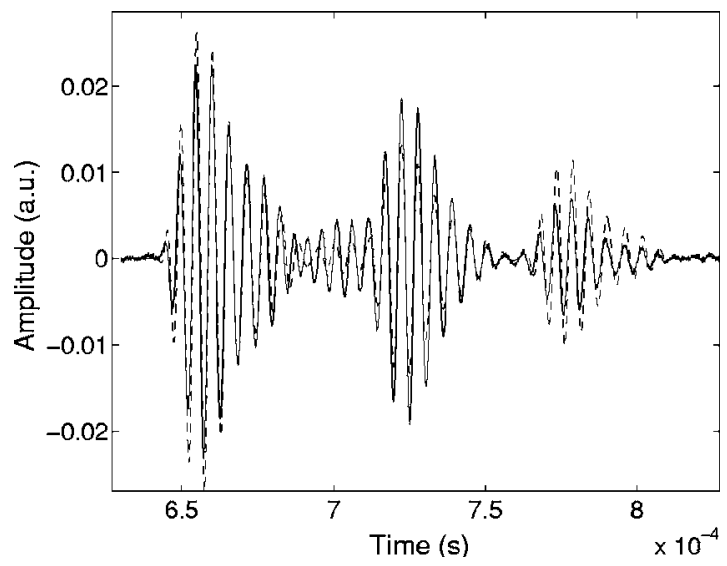

FIG. 11. Comparison between experimental reflected signal (solid line) and simulated reflected signal (dashed line) for a double-layered medium consisting of $1.11 \mathrm{~cm}$ of M4 and $0.87 \mathrm{~cm}$ of M1.

$$
\left(\begin{array}{l}
A_{2}(z) \\
B_{2}(z)
\end{array}\right)=\frac{1}{2}\left(\begin{array}{cc}
1 & -1 \\
1 & 1
\end{array}\right)\left(\begin{array}{c}
1+R(z) \\
K_{1}(R(z)-1)
\end{array}\right) .
$$

From Eqs. (27) and (33), one has

$$
\left(\begin{array}{l}
A_{3}(z) \\
B_{3}(z)
\end{array}\right)=\frac{T^{\prime}(z)}{2}\left(\begin{array}{c}
\left(1+K_{3}\right) \exp \left(\frac{\sqrt{f_{3}(z)}}{c_{3}}(\ell+L)\right) \\
\left(1-K_{3}\right) \exp \left(-\frac{\sqrt{f_{3}(z)}}{c_{3}}(\ell+L)\right)
\end{array}\right) \text {. }
$$

where $T^{\prime}(z)=T(z) \exp \left\{-\left[\left(\ell / c_{2}\right)+\left(L / c_{3}\right)\right] z\right\}$.

Using Eqs. (46),(47), and Eqs. (26) and (32), we obtain the following linear system given the reflection and transmission coefficients $R(z)$ and $T(z)$ :

$$
\begin{gathered}
R\left[\cosh \left(\frac{\sqrt{f_{2}(z)}}{c_{2}} \ell\right)+K_{1} \sinh \left(\frac{\sqrt{f_{2}(z)}}{c_{2}} \ell\right)\right]-T^{\prime}(z) \\
\quad \times\left[\cosh \left(\frac{\sqrt{f_{3}(z)}}{c_{3}} L\right)+K_{3} \sinh \left(\frac{\sqrt{f_{3}(z)}}{c_{3}} L\right)\right] \\
=K_{1} \sinh \left(\frac{\sqrt{f_{2}(z)}}{c_{2}} \ell\right)-\cosh \left(\frac{\sqrt{f_{2}(z)}}{c_{2}} \ell\right)
\end{gathered}
$$

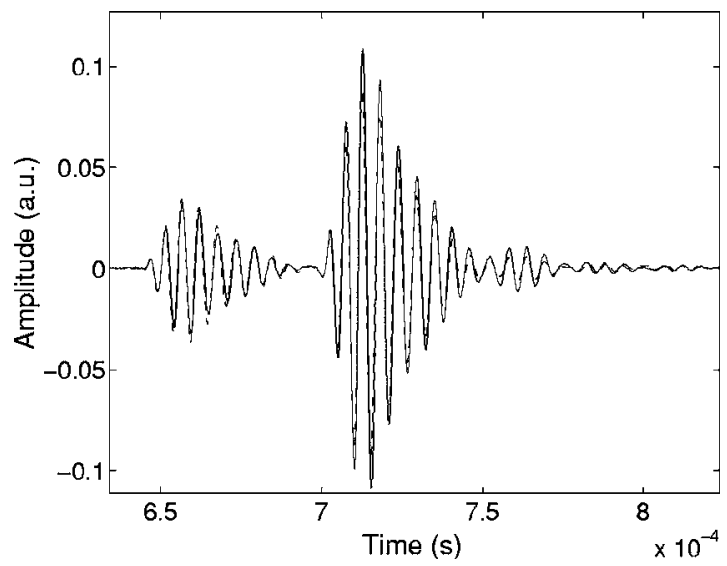

FIG. 12. Comparison between experimental reflected signal (solid line) and simulated reflected signal (dashed line) for a double-layered medium consisting of $0.88 \mathrm{~cm}$ of $\mathrm{M} 1$ and $0.83 \mathrm{~cm}$ of M2.

$$
\begin{gathered}
R\left[K_{2} \sinh \left(\frac{\sqrt{f_{2}(z)}}{c_{3}} \ell\right)+K_{3} \cosh \left(\frac{\sqrt{f_{2}(z)}}{c_{2}} \ell\right)\right]+T^{\prime}(z) \\
\quad \times\left[\sinh \left(\frac{\sqrt{f_{3}(z)}}{c_{3}} L\right)+K_{3} \cosh \left(\frac{\sqrt{f_{3}(z)}}{c_{3}} L\right)\right] \\
=K_{3} \cosh \left(\frac{\sqrt{f_{2}(z)}}{c_{2}} \ell\right)-K_{2} \sinh \left(\frac{\sqrt{f_{2}(z)}}{c_{2}} \ell\right) .
\end{gathered}
$$

By setting

$$
\begin{aligned}
D(z)= & \left(1+K_{3}^{2}\right) \cosh \left(\frac{\sqrt{f_{2}(z)}}{c_{2}} \ell\right) \sinh \left(\frac{\sqrt{f_{3}(z)}}{c_{3}} L\right) \\
& +\left(K_{1}+K_{2} K_{3}\right) \sinh \left(\frac{\sqrt{f_{2}(z)}}{c_{2}} \ell\right) \sinh \left(\frac{\sqrt{f_{3}(z)}}{c_{3}} L\right) \\
& +2 K_{3} \cosh \left(\frac{\sqrt{f_{2}(z)}}{c_{2}} \ell\right) \cosh \left(\frac{\sqrt{f_{3}(z)}}{c_{3}} L\right) \\
& +\left(K_{2}+K_{1} K_{3}\right) \sinh \left(\frac{\sqrt{f_{2}(z)}}{c_{2}} \ell\right) \cosh \left(\frac{\sqrt{f_{3}(z)}}{c_{3}} L\right),
\end{aligned}
$$

and

$$
\begin{aligned}
D_{1}(z)= & \left(K_{1}-K_{2} K_{3}\right) \sinh \left(\frac{\sqrt{f_{2}(z)}}{c_{2}} \ell\right) \sinh \left(\frac{\sqrt{f_{3}(z)}}{c_{3}} L\right) \\
& +\left(K_{3}^{2}-1\right) \cosh \left(\frac{\sqrt{f_{2}(z)}}{c_{2}} \ell\right) \sinh \left(\frac{\sqrt{f_{3}(z)}}{c_{3}} L\right) \\
& +\left(K_{1} K_{3}-K_{2}\right) \sinh \left(\frac{\sqrt{f_{2}(z)}}{c_{2}} \ell\right) \cosh \left(\frac{\sqrt{f_{3}(z)}}{c_{3}} L\right),
\end{aligned}
$$

one has the following expressions for $R(z)$ and $T(z)$ :

$$
R=\frac{D_{1}(z)}{D(z)} \quad \text { and } \quad T^{\prime}(z)=\frac{2 K_{1} K_{2}}{D(z)}
$$

which are equivalent to the expressions (35) and (36) given in Sec. IV.

\section{APPENDIX B: GREEN FUNCTION OF THE MEDIUM}

The Green function ${ }^{28} F_{i}, i=2,3$ of the porous layer (2) and (3), respectively is given by

$F_{i}(t, k)$

$$
= \begin{cases}0 & \text { if } 0 \leqslant t \leqslant k \\ \mathfrak{J}_{i}(t)+\Delta_{i} \int_{0}^{t-k} \varnothing_{i}(t, \xi) d \xi, & \text { if } t \geqslant k, \quad i=2,3\end{cases}
$$

with

$$
\mathfrak{J}_{i}(t)=\frac{\psi_{i}}{4 \sqrt{\pi}} \frac{k}{(t-k)^{3 / 2}} \exp \left(-\frac{\psi_{i}^{2} k^{2}}{16(t-k)}\right), \quad i=2,3,
$$

where $\varnothing_{i}(\tau, \xi), i=2,3$ has the following form: 


$$
\begin{aligned}
\varnothing_{i}(\xi, \tau)= & -\frac{1}{4 \pi^{3 / 2}} \frac{k}{\sqrt{(\tau-\xi)^{2}-k^{2}}} \frac{1}{\xi^{3 / 2}} \\
& \times \int_{-1}^{1} \exp \left(-\frac{\mathfrak{N}_{i}(\mu, \tau, \xi)}{2}\right)\left(\mathfrak{N}_{i}(\mu, \tau, \xi)-1\right) \\
& \times \frac{\mu d \mu}{\sqrt{1-\mu^{2}}}, \quad i=2,3,
\end{aligned}
$$

and where

$$
\begin{aligned}
& \mathfrak{N}_{i}(\mu, \tau, \xi)=\left(\Delta_{i} \mu \sqrt{(\tau-\xi)^{2}-k^{2}}+\psi_{i}(\tau-\xi)\right)^{2} / 8 \xi \\
& \psi_{i}=2 \alpha_{i} \sqrt{\frac{\eta}{\pi}}\left(\frac{1}{\Lambda_{i}}+\frac{\gamma-1}{\sqrt{P_{r}} \Lambda_{i}^{\prime}}\right), \quad \varphi_{i}=\frac{4 \alpha_{i}(\gamma-1) \eta}{\Lambda_{i} \Lambda_{i}^{\prime} \sqrt{P_{r}} \rho_{f}} \\
& \Delta_{i}^{2}=\psi_{i}^{2}-4 \varphi_{i}, \quad i=2,3 .
\end{aligned}
$$

when $k \rightarrow \infty, \mathfrak{J}_{i}$ and $\varnothing_{i}(\xi, \tau)$ tends to zero, then the Green function $F_{i}(t, k)$ also tends to zero.

${ }^{1}$ J. F. Allard, Propagation of Sound in Porous Media: Modeling Sound Absorbing Materials (Chapman and Hall, London, 1993).

${ }^{2} \mathrm{~K}$. Attenbourough, "On the acoustic slow wave in air-filled granular media," J. Acoust. Soc. Am. 81, 93-102 (1986).

${ }^{3}$ P. Leclaire, L. Kelders, W. Lauriks, N. R. Brown, M. Melon, and B. Castagnède, "Determination of the viscous and thermal characteristics lengths of plastic foams by ultrasonic measurements in helium and air," J. Appl. Phys. 80, 2009-2012 (1996).

${ }^{4}$ P. Leclaire, L. Kelders, W. Lauriks, C. Glorieux, and J. Thoen, "Determination of the viscous characteristic length in air-filled porous materials by ultrasonic attenuation measurements," J. Acoust. Soc. Am. 99, 1944-1948 (1996).

${ }^{5}$ G. Caviglia and A. Morro, "A closed-form solution for reflection and transmission of transient waves in multilayers," J. Acoust. Soc. Am. 116, 643-654 (2004).

${ }^{6}$ G. V. Norton and J. C. Novarini, "Including dispersion and attenuation directly in time domain for wave propagation in isotropic media," $\mathrm{J}$. Acoust. Soc. Am. 113, 3024-3031 (2003).

${ }^{7}$ W. Chen and S. Holm, "Modified Szabo's wave equation models for lossy media obeying frequency power law," J. Acoust. Soc. Am. 113, 30243031 (2003).

${ }^{8}$ Z. E. A. Fellah, M. Fellah, W. Lauriks, and C. Depollier, "Direct and inverse scattering of transient acoustic waves by a slab of rigid porous material," J. Acoust. Soc. Am. 114, 2570-2574 (2003).

${ }^{9}$ Z. E. A. Fellah, C. Depollier, S. Berger, W. Lauriks, P. Trompette, and J. Y. Chapelon, "Determination of transport parameters in air-saturated porous materials via reflected ultrasonic waves," J. Acoust. Soc. Am. 114(5), 2561-2569 (2003).

${ }^{10}$ Z. E. A. Fellah, F. G. Mitri, C. Depollier, S. Berger, W. Lauriks, and J. Y. Chapelon, "Characterization of porous materials with a rigid frame via reflected waves," J. Appl. Phys. 94, 7914-7922 (2003).
${ }^{11}$ Z. E. A. Fellah, S. Berger, W. Lauriks, and C. Depoller, "Verification of Kramers-Kronig relationships in porous materials having a rigid frame," J. Sound Vib. 270, 865-885 (2004).

${ }^{12}$ T. L. Szabo, "Time domain wave equations for lossy media obeying a frequency power law," J. Acoust. Soc. Am. 96, 491-500 (1994).

${ }^{13}$ T. L. Szabo, "Causal theories and data for acoustic attenuation obeying a frequency power law,” J. Acoust. Soc. Am. 97, 14-24 (1995).

${ }^{14}$ Inverse Problems in Mathematical Physics, edited by L. Päivärinta and E. Somersalo (Springer, Berlin, 1993).

${ }^{15}$ F. Mainardi, "Transient waves in linear viscoelasticity," in Vibration and Control of Structures, edited by A. Gurran (World Scientific, Singapore, 1997).

${ }^{16}$ D. L. Johnson, J. Koplik, and R. Dashen, "Theory of dynamic permeability and tortuosity in fluid-saturated porous media," J. Fluid Mech. 176, 379-402 (1987).

${ }^{17}$ W. Sachse and Y. H. Pao, "On the determination of phase and group velocities of dispersive waves in solids," J. Appl. Phys. 49, 4320-4327 (1978).

${ }^{18} \mathrm{~K}$. V. Gurumurthy and R. M. Arthur, "A dispersive model for the propagation of ultrasound in soft tissue," Ultrason. Imaging 49, 355-377 (1982).

${ }^{19} \mathrm{R}$. Kuc, "Modeling acoustic attenuation of soft tissue with a minimumphase filter," Ultrason. Imaging 6, 24-36 (1984).

${ }^{20} \mathrm{~F}$. Yu, A. Rossikhin, and M. V. Shitikova, "Application of fractional calculus to dynamic problems of linear hereditary mechanics of solids," Appl. Mech. Rev. 50, 15-67 (1997).

${ }^{21}$ M. Caputo, "Vibration of an infinite plate with a frequency dependent Q," J. Acoust. Soc. Am. 60, 634-639 (1976).

${ }^{22}$ R. L. Bagley and P. J. Torvik, "On the fractional calculus model of viscoelastic behavior,” J. Rheol. 30, 133-155 (1986).

${ }^{23}$ A. Hanyga and V. E. Rok, "Wave propagation in micro-heterogeneous porous media: A model based on an integro-differential wave equation," J. Acoust. Soc. Am. 107, 2965-2972 (2000).

${ }^{24} \mathrm{M}$. A. Biot, "The theory of propagation of elastic waves in fluid-saturated porous solid. I. Low frequency range," J. Acoust. Soc. Am. 28, 168-178 (1956).

${ }^{25}$ M. A. Biot, "The theory of propagation of elastic waves in fluid-saturated porous solid. II. Higher frequency range," J. Acoust. Soc. Am. 28, 179191 (1956).

${ }^{26}$ Z. E. A. Fellah and C. Depollier, "Transient wave propagation in rigid porous media: A time domain approach," J. Acoust. Soc. Am. 107, 683688 (2000).

${ }^{27}$ S. G. Samko, A. A. Kilbas, and O. I. Marichev, Fractional Integrals and Derivatives: Theory and Applications (Gordon and Breach Science, Amsterdam, 1993).

${ }^{28}$ Z. E. A. Fellah, M. Fellah, W. Lauriks, C. Depollier, J. Y. Chapelon, and Y. C. Angel, "Solution in time domain of ultrasonic propagation equation in a porous material," Wave Motion 38, 151-163 (2003).

${ }^{29}$ Z. E. A. Fellah, S. Berger, W. Lauriks, C. Depollier, C. Aristegui, and J. Y. Chapelon, "Measuring the porosity and tortuosity of porous materials via reflected waves at oblique incidence," J. Acoust. Soc. Am. 113(5), 24242433 (2003).

${ }^{30}$ Z. E. A. Fellah, S. Berger, W. Lauriks, C. Depollier, and M. Fellah, "Measuring the porosity of porous material having rigid frame via reflected waves: A time domain analysis with fractional derivatives," J. Appl. Phys. 93, 296-303 (2003). 